\title{
In-situ characterization of interface delamination by a new miniature mixed mode bending setup
}

\author{
M. Kolluri - M. H. L. Thissen • \\ J. P. M. Hoefnagels • J. A. W. van Dommelen • \\ M. G. D. Geers
}

Received: 14 November 2008 / Accepted: 20 April 2009 / Published online: 4 June 2009

(C) The Author(s) 2009. This article is published with open access at Springerlink.com

\begin{abstract}
A new miniature mixed mode bending (MMMB) setup for in-situ characterization of interface delamination in miniature multi-layer structures was designed and realized. This setup consists of a novel test configuration to accomplish the full range of mode mixities and was specially designed with sufficiently small dimensions to fit in the chamber of a scanning electron microscope (SEM) or under an optical microscope for detailed real-time fracture analysis during delamination. Special care was taken to minimize the effects of friction, the influence of gravity, and non-linearities due to the geometry of the setup. The performance of the setup was assessed using specially-designed test samples supported by finite element analyses. Delamination experiments conducted on homogeneous bilayer samples in mode I and mixed mode loading were visualized with a scanning electron microscope and showed the formation of small micro cracks ahead of the crack tip followed by crack bridging and a full crack, thereby demonstrating the advantages of in-situ testing to reveal the microscopic delamination mechanism.
\end{abstract}

M. Kolluri

Materials Innovation Institute (M2i), Mekelweg 2,

P.O. Box 5008, 2600 GA Delft, The Netherlands

M. Kolluri · M. H. L. Thissen · J. P. M. Hoefnagels ( $\varangle)$.

J. A. W. van Dommelen · M. G. D. Geers

Department of Mechanical Engineering, Eindhoven

University of Technology, W.H. 4.13, P.O. Box 513,

$5600 \mathrm{MB}$ Eindhoven, The Netherlands

e-mail: j.p.m.hoefnagels@tue.nl
Keywords Delamination - Interface mechanics $\cdot$ Mode mixity $\cdot$ Miniaturization . In-situ characterization

\section{Introduction}

The demand for the continuous miniaturization and multi-functionality of electronic devices led the electronic industry towards new packaging technologies like "System in Package" (SiP). SiP technology primarily involves assembly of several components (active components, passive components and MEMS) into a single package to achieve different functionalities. Consequently, SIP-microsystems contain several interfaces formed between stacked, multiple, thin layers, manufactured using different materials and processes. Interfaces are often recognized as critical regions for the reliability of these products because the presence of high stresses at these interfaces (e.g. due to a mismatch in coefficient of thermal expansion (CTE) and Poisson's ratio) and in-plane shear stresses often lead to delamination (Hutchinson and Suo 1992; Evans and Hutchinson 1995; Dauskardt et al. 1998; Vlassak et al. 2005). Much research is ongoing to improve the interface behavior by adopting suitable coatings or cleaning techniques at the interface during manufacturing of these components (Srikanth et al. 2006). However, detailed quantitative characterization of the interfaces in these systems is required in order to improve design 
rules for manufacturing and improve reliability during service of these products.

A number of experimental techniques have been developed and reported in the literature (e.g. Evans et al. 1990; Dreier et al. 1992; Gupta and Pronin 1995; Volinsky et al. 2003; Li and Siegmund 2004; Banks-Sills et al. 2005; Wang et al. 2006; Ocaña et al. 2006; Liu et al. 2008) to measure interfacial properties, most of which use a fracture mechanics approach because of its proven versatility. A fracture mechanics approach requires an experimental setup capable of triggering delamination and uses the resulting force-displacement data to obtain the interface fracture toughness or critical energy release rate (CERR) as an important interface parameter to characterize the interface. It has been shown by many authors (e.g. Hu and Evans 1989; Hutchinson and Suo 1992; Banks-Sills et al. 2000; Kuhl and Qu 2000; Shi et al. 2006; Tang et al. 2007) that the CERR for crack propagation varies significantly with mode mixity. Furthermore, the presence of dissimilar materials forming the interface, which is common in microsystems, increases the complexity of the CERR measurement. This indicates that the experimental technique to characterize an interface system should be able to measure the CERR over the entire range of mode mixities, in order to identify valid input parameters in a design limit criterion. Techniques reported in the literature to measure the CERR include the well-known double cantilever beam (DCB) test for pure mode-I loading (ASTM D 5528-01, 2001) and end notch flexure (ENF) test for applying pure mode-II loading (Carlsson et al. 1986), as well as mixed mode bending (MMB) setups (e.g. Reeder and Crews 1990; Soboyejo et al. 1999; Merrill and Ho 2004; Blanco et al. 2006; Thijsse et al. 2008), which cover a range of mode mixities, see Fig. 1. Of all setups, only the MMB setups yield the CERR over a full range of mode mixities with a single test configuration.

The prime difficulty in many of the existing delamination experiments is the identification of the crack tip location in order to trace the crack length, which is needed to calculate the fracture toughness. To circumvent this problem, critical loads deduced from experiments, where the delamination starts for a given pre-crack length, were used to calculate the CERR with the aid of analytical formulae (Davidson and Sundararaman 1996; Davies et al. 1998; Soboyejo et al. 1999). Stiffness lines generated with finite element (FE) models of known crack length specimens were used to evaluate the CERR by (Thijsse et al. 2008). Some authors (e.g. Vinciquerra and Davidson 2004; Gunderson et al. 2007) reported that a crack length measurement with a simple optical magnification lens system may be erroneous and also highlighted the need for techniques which can measure crack lengths more accurately. Furthermore, detailed in-situ visualization of the delamination process is essential to accurately pin-point the crack tip location, to measure quantitative delamination characteristics such as the crack opening profile and the process zone size, and to obtain more insight in the fracture events along the interface.

In-situ characterization in a microscope, however, puts serious constraints on the overall size of the delamination setup. Evaluation of existing MMB setups elucidates the difficulties to use them for in-situ testing. In case of the setup of Reeder and Crews (1990), shown in Fig. 2a, restricted dimensions of the design space prevent the lever from having sufficient length to cover the complete range of mode mixities. Furthermore, this test is difficult to perform in the horizontal plane (i.e. keeping the direction of load application in the horizontal plane), which is necessary to enable the use of standard microscopes to trace the crack tip movement during in-situ delamination testing, (e.g. the viewing axis of a scanning electron microscope (SEM) is always in the vertical direction). Merrill and Ho's setup (Merrill and Ho 2004), shown in Fig. 2b, was also constructed such that the loading direction is vertical, leading to similar limitations. Furthermore, gravity acts on both of the above mentioned setups, resulting in additional undesired forces on the sample. In the setup of Thijsse et al. (2008), a counter balance was added to the loading configuration of Reeder and Crews (1990) to minimize the influence of gravity. However, a persistent conse-
Fig. 1 Sketch of different loading configurations for interface delamination. a DCB; b ENF; c Mixed mode bending

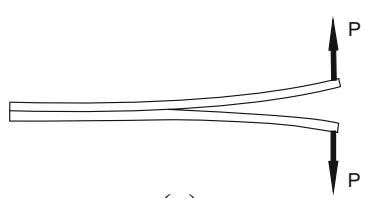

(a)

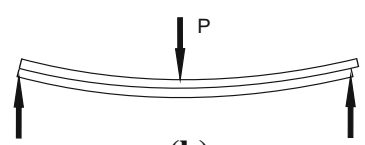

(b)

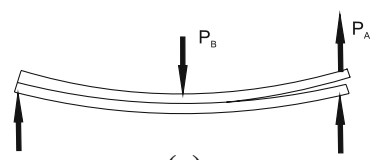

(c) 
Fig. 2 Two different existing concepts for mixed mode delamination testing. a Reeder and Crews (1990); b Merrill and Ho (2004)

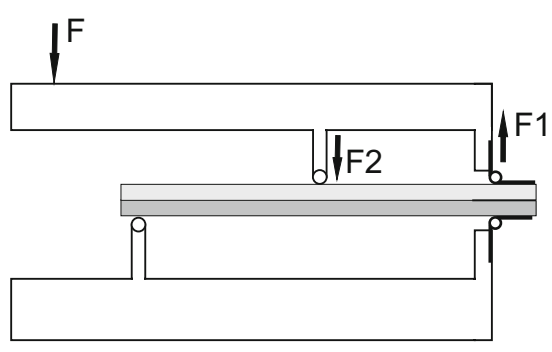

(a)

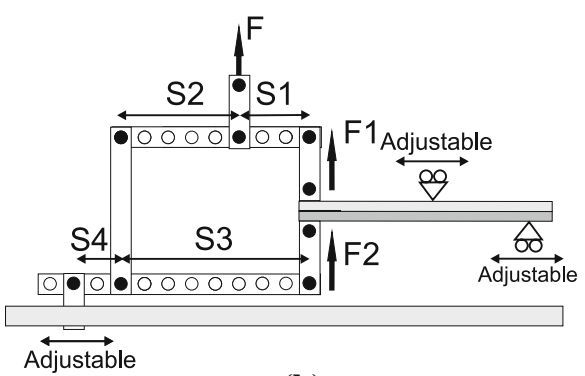

(b) quence of gravity is a strong dependence of the mode angle on the crack length. Finally, all currently available methods are hampered by frictional non-linearities in hinges that are attached to the sample and rotation points/joints in the loading frame.

The present work focuses on the design and realization of a miniaturized mixed mode bending setup which enables in-situ delamination testing for the full range of mode mixities. This setup is designed such that effects of friction, gravity, and geometrical non-linearities are minimized. The performance of the setup is assessed using specially-designed test samples supported by FE analyses. In-situ delamination experiments performed on homogeneous bilayer samples are presented to prove the functionality of the setup and illustrate its capabilities for interfacial characterization.

\section{Design of the miniaturized mixed mode bending (MMMB) apparatus}

\subsection{New configuration for mixed mode loading}

A key constraint in the design of the new setup is its size, which should be small enough (i) to handle multi-layer structures that are representative for stacked layers present in SiPs and (ii) to be mounted in a commercially available micro tensile stage (Kammrath \& Weiss $\mathrm{GmbH}$, with an available design space of $55 \times 47 \times 29 \mathrm{~mm}$ ) which in turn fits in the chamber of a SEM for in-situ delamination testing. Simple down scaling of existing MMB setups is not feasible because of the incompatible load frame configuration and the sample orientation that prevents in-situ microscopic observation. Therefore, a new test configuration was developed that meets the above mentioned requirements and realizes the MMB loading as shown in Fig. 1c. The MMB configuration is preferred because
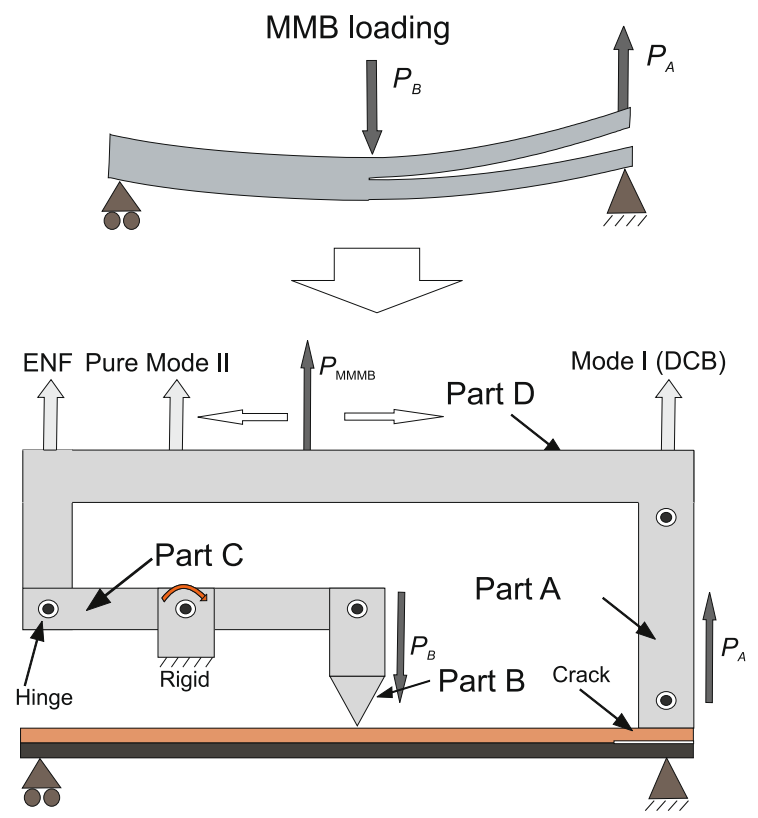

Fig. 3 Schematic representation of the new loading geometry for mixed mode bending

it is standardized (ASTM D6671-01, 2001) and generally accepted for characterization of interfacial delamination. A schematic representation of the new loading geometry of the MMMB apparatus is depicted in Fig. 3.

The setup consists of four rigid parts (A to D in Fig. 3) connected with hinges. Advantages of the present design are: (i) its loading mechanism that allows to access the maximum range of interfacial loading modes, from double cantilever bending (pure mode I delamination), to pure mode II delamination, to end notch flexure in a single setup; (ii) its compact geometry (allowing it to be used in the chamber of an electron microscope); (iii) the insensitivity of its force measurements to its self weight, because the loading of the sample is done in the horizontal plane. 
Fig. 4 Schematic representation of the load distribution in mixed mode bending with the loads on the sample (left) and the decomposition of these loads into mode I and mode II components (right)

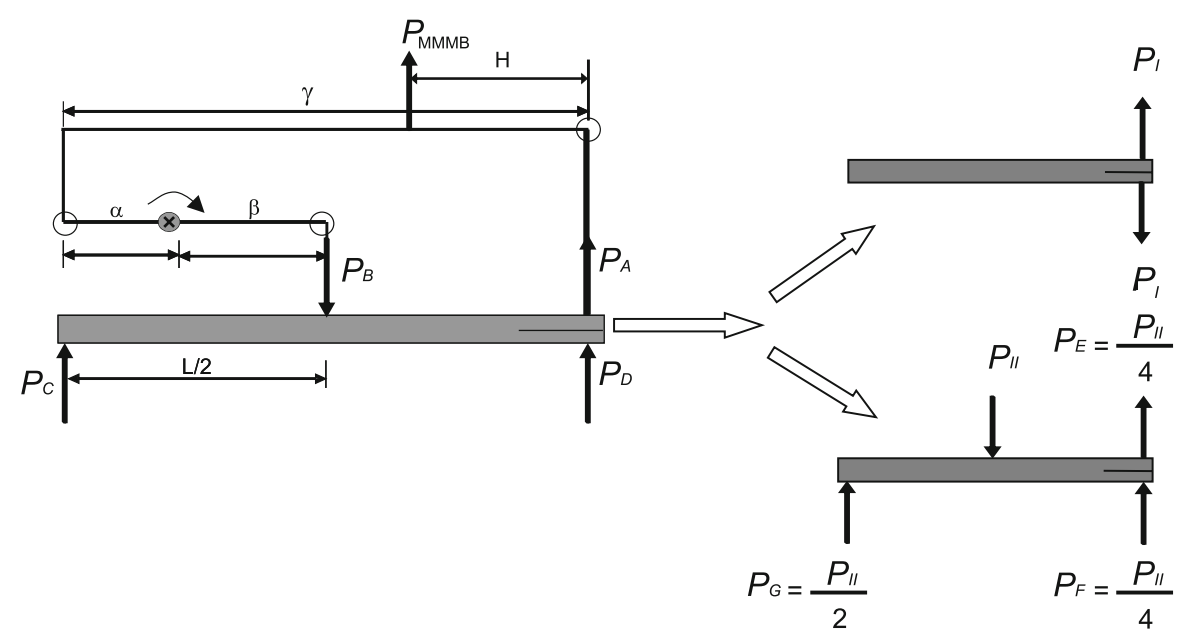

The mixed mode bending loads are applied by a new lever mechanism. Part ' $C$ ' is pinned to the outside world allowing it only to rotate in testing plane. By the application of a force $P_{\mathrm{MMMB}}$ in a certain position on part 'D', part 'B' moves downward while part 'A' moves upward, generating two oppositely directed forces $P_{A}$ and $P_{B}$ on the sample. The ratio of the forces $P_{A}$ and $P_{B}$ depends on the position of the loading point on part ' $\mathrm{D}$ ', triggering different loading modes as discussed in more detail below.

An analysis of the loads applied to the sample in the new test setup shows that the loads transferred to the sample, which are depicted in the left hand side of Fig. 4, can be written as:

$$
\begin{aligned}
P_{A} & =P_{\text {MMmB }}(1-\xi) \\
P_{B} & =P_{\text {MMMB }} \frac{\alpha}{\beta} \xi \\
P_{C} & =\frac{P_{B}}{2} \\
P_{D} & =-P_{A}+\frac{P_{B}}{2},
\end{aligned}
$$

where, $\xi=\frac{H}{\gamma}$ is a dimensionless shape parameter which represents the relative position of the applied load, $H$ is the corresponding absolute position and $\alpha, \beta, \gamma$ are the characteristic dimensions of the loading mechanism. Finally, $P_{\text {MMMB }}$ is the actual value of the applied mixed mode bending load.

The load on the sample can be decomposed in its mode I and pure mode II components, $P_{I}$ and $P_{I I}$, respectively, which are defined by the load configurations depicted in the top and bottom right hand side of Fig. 4. The corresponding expressions for these mode I and mode II components are:

$$
\begin{aligned}
& P_{I}=\frac{P_{A}+P_{D}}{2}=P_{\text {MMMB }}\left(1-\xi-\frac{\alpha}{4 \beta} \xi\right) \\
& P_{I I}=P_{B}=P_{\text {MMMB }} \frac{\alpha}{\beta} \xi .
\end{aligned}
$$

Pure mode II loading is defined such that the two loads $P_{E}$ and $P_{F}$ acting on the top and bottom arms of the specimen in the pre-cracked region (see bottom right of Fig. 4) are equal in order to have a zero mode I component on the interface. It is clear from the above analysis that when the load is applied at the right hand side of the upper lever (part 'D'), i.e. when $\xi=0$, the applied loading corresponds to pure mode I loading:

$P_{I}=P_{A}=P_{\mathrm{MMMB}} \quad$ and $P_{I I}=0$. for $\xi=0$.

In the other extreme, when the load is applied at the left end side of the upper lever (part 'D'), i.e. when $\xi=1$, the applied load resembles conventional ENF loading, i.e.

$P_{I I}=P_{B}, \quad$ but $P_{I}=-P_{\mathrm{MMMB}} \frac{\alpha}{4 \beta}$. for $\xi=1$.

Since in this case $P_{I} \neq 0$, this ENF test can be considered as a combined mode II and compressive mode I test. This compressive mode I component may cause friction between the two contacting layers. Therefore, the End Notch Flexure (ENF) case, which is frequently used for mode II fracture analysis, does not represent a pure mode II test. The resulting friction leads to an overestimation of the interface toughness in the experiments. Several studies (Davies 1997; Fan et al. 2007; Bing and Sun 2007) also highlighted this problem due to friction when the ENF test is used to determine the mode II delamination resistance. However, a position 
for which the mode I component is zero and consequently a pure mode II loading is obtained can be identified in the new loading geometry. This position is given by:

$$
\xi=\frac{4 \beta}{\alpha+4 \beta} \text {. }
$$

\subsection{Design of the test apparatus}

The new configuration, shown in the previous section, consists of a mechanism with several moving parts and hinges. The use of conventional hinges commonly introduces hysteresis in the force measurements because of the non-negligible frictional dissipation during rotation at these hinges, particularly in a miniaturized setup. To avoid this hysteresis in the test apparatus, specially designed elastic hinges were used. Figure 5 shows an FE model of the elastic hinge used in the present setup in a deformed state. Parameters defining the geometry are also shown in the figure, as well as a safety element that is used to limit the rotation angle $\phi$ of the hinge to the maximum achievable rotation, $\phi_{m}$, to prevent permanent deformations in the central bridge of the hinge. $\phi_{m}$ is determined by the material properties, for a given geometry of the hinge. A Ti-6Al$4 \mathrm{~V}$ alloy was used for the current setup because of its

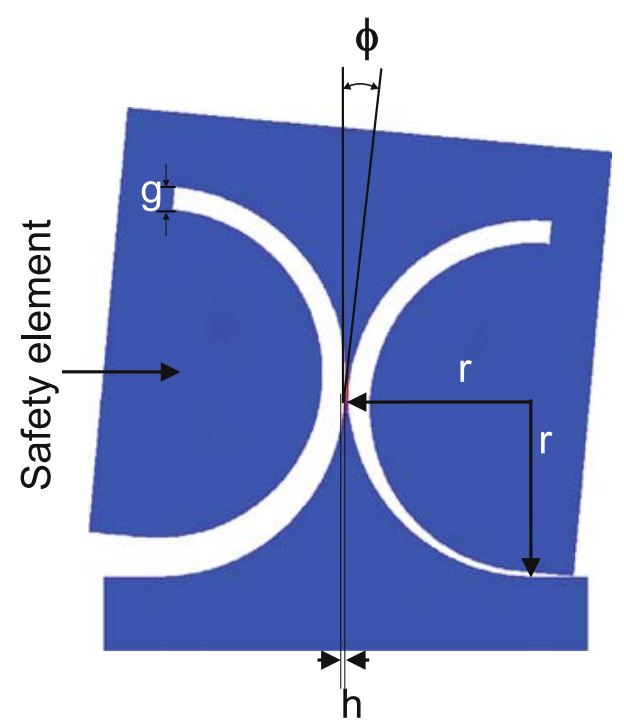

Fig. 5 Finite element model of the elastic hinge used in the current setup in a deformed state. Parameters defining the geometry $(r$ : radius, $h$ : width of the bridge and $g$ : width of the safety groove) are also shown favorable combination of yield strength and Young's modulus. Further details about the design of such elastic hinges can be found in Smith et al. (1997). In total, the setup contains 8 elastic hinges, each with dimensions of $r=2.5 \mathrm{~mm}, h=50 \mu \mathrm{m}, g=0.3 \mathrm{~mm}$ and $t=2 \mathrm{~mm}$ and a maximum rotation $\phi=5.2^{\circ}$, allowing for an axial load of $20 \mathrm{~N}$, which was verified through FE simulations.

The main parts of the device are depicted in Fig. 6. The 'Main Loading Mechanism', MLM, is connected to the 'Position bar' with the so called 'Mode selector'. To change the applied mode angle, the Mode selector can be placed at various discrete positions on the MLM using dovetail connectors. The arrow on the 'position bar' indicates the fixed direction and position of the externally applied force. Two 'dovetail connectors' attached on both sides of the sample are used to attach the sample to the MLM and the support hinge. The support hinge connects the sample to the real world leaving only one rotational degree of freedom. Figure 7 shows a picture of the whole setup placed inside a micro tensile stage. The range of sample dimensions which can fit in the MMMB device are (length $\times$ width $\times$ height): 35 $\times 1-7.5 \times 0.5-6 \mathrm{~mm}$.

Other elements in the setup design include (i) an adjustment mechanism to adapt the setup to a certain sample height (part 9 and 11 in Fig. 7), (ii) a screw mechanism (part 10 in Fig. 7) to adjust the alignment of the sample, and (iii) different details to overcome out-of-plane deflections in the device and to avoid friction between the moving parts and the rigid bottom plate. The latter is achieved by two flexible pins positioned in vertical direction, which support the system and increase its stability.

\subsection{Finite element analysis}

The MMMB setup with a mounted homogeneous bilayer sample has been modeled in a finite element program (MSC.Marc/Mentat), see Fig. 8. In total, the sample is described with 32,856 eight-node quadrilateral elements and the MLM is modeled with 52,196 four-node quadrilateral elements. A predefined crack was present in the bilayer sample, with a special crack tip mesh which has a rosette shape with transition elements and quarter-point elements. Frictionless contact conditions were used between the two surfaces of the cracked region of the specimen. The material properties 
Fig. 6 a Design of the new MMMB device and b picture of the real device: 1: Main Loading Mechanism (MLM), 2: Mode selector, 3: Sample, 4: Support, 5: Support hinge, 6: Dovetail connector, 7: Position bar, 8: Loading tip 9: Setscrew for sample height, 10:

Setscrew for alignment, 11: Sample height adjuster, 12: Protective plate

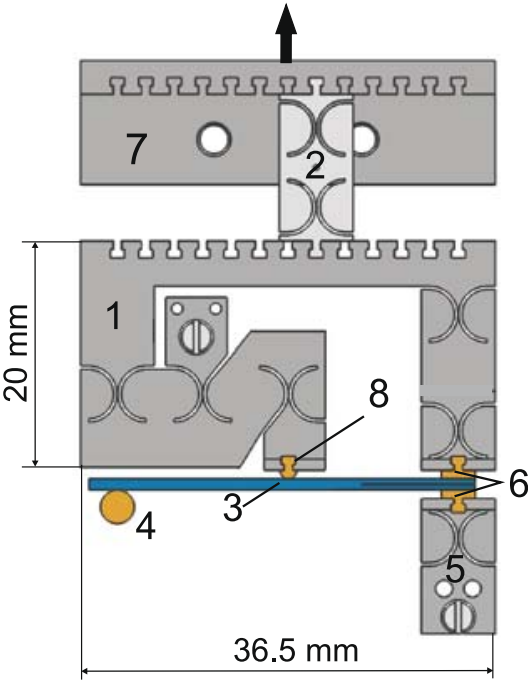

(a)

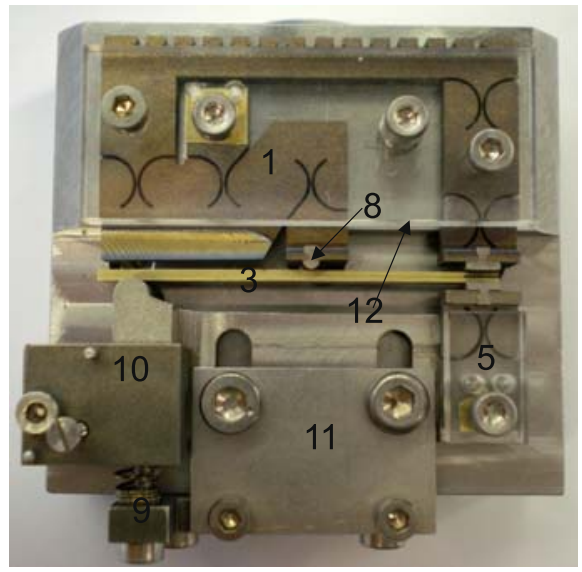

(b)
Fig. 7 MMMB device, mounted in a micro tensile stage, with: 1: Main Loading Mechanism (MLM), 2: Mode selector, 3: Sample, 5: Support hinge, 7: Position bar, 9: Setscrew for sample height, 10: Setscrew for alignment, 11: Sample height adjuster, 13: Micro tensile stage, 14: Load cell

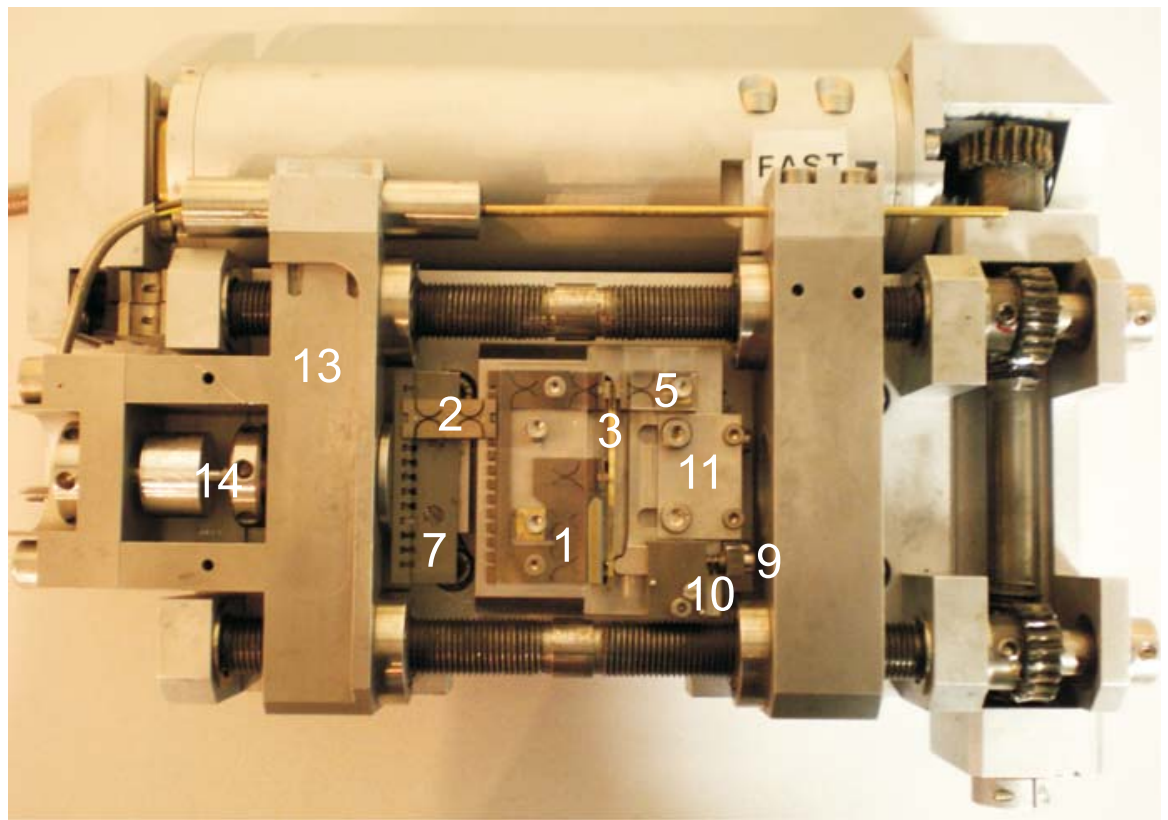

used for the sample and the MLM are given in Table 1. Simulations were performed by assuming plane strain conditions and linear elastic material behavior.

To assess the behavior of the setup over a complete range of mode mixities, simulations were performed by applying the load $\left(P_{\mathrm{MMMB}}\right)$ at different load application positions ( $\xi$ ranging from 0 to 1 ) for a specimen with various fixed crack lengths. Mode angles, $\psi$, were calculated from the stress profiles ahead of the crack tip using, $\psi=\arctan \left(\frac{\sigma_{12}}{\sigma_{22}}\right)_{\delta}$,

where $\sigma_{22}$ is the normal stress, $\sigma_{12}$ is the shear stress and $\delta$ is a characteristic distance from the crack tip along the interface. This distance needs to be tuned in case of interfaces between dissimilar materials (Suo and Hutchinson 1990), because of the existence of a complex stress state at those interfaces. In case of homogeneous bilayer samples, $\delta$ can be any value within the K-dominance region, and therefore $\delta=50 \mu \mathrm{m}$ was 
Fig. 8 a FE model of the setup and sample with boundary conditions and $\mathbf{b}$ Magnified view of an elastic hinge (indicated with a rectangle in a)

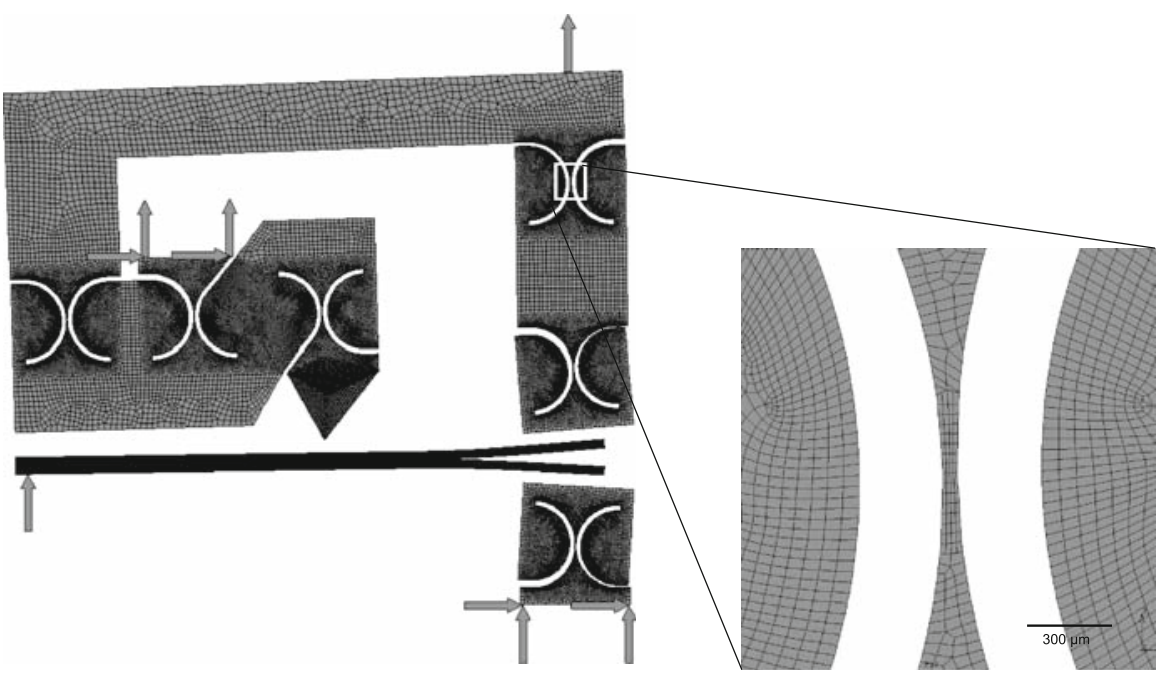

(b) chosen in the present analysis. The mode angles, calculated from the simulation results of a specimen with a $6 \mathrm{~mm}$ pre-crack length at $400 \mu \mathrm{m}$ displacement applied to the main loading mechanism (MLM displacement), are shown as a function of loading position $\xi$ in Fig. 9a. This figure shows that the new MMMB setup permits to access the complete range of mode mixities from $0^{\circ}$ to $90^{\circ}$ mode angle by changing the loading position $\xi$ from 0 to 0.8 , respectively. The mode angle is about $90^{\circ}$ for all positions in the range $\xi=0.8-1$. As shown in the previous section, pure mode II loading is obtained with a loading position $\xi=\frac{4 \beta}{\alpha+4 \beta}$, which, in the present setup, equals $\xi=0.8$. When the load is applied at positions $\xi \geq 0.8$, an additional compressive mode I component exists (Eq. 2) in addition to the pure mode II component acting at the crack tip. The presence of a compressive mode I component acting on the extremity of the specimen does not significantly influence the mode angle obtained from the stress field ahead of the crack tip. Note, however, that delamination experiments performed at those positions are not completely representative for pure mode II conditions, since the friction induced at the fractured interface may influence the delamination behaviour. This also applies to conventional ENF tests, which are widely used as a Mode II delamination test. Implications of these differences are discussed in the results and discussion section.

The FE model was also used to evaluate the influence of the applied MLM displacement and the crack length on the mode angle. In Fig. 9b, the mode angle is shown as a function of MLM displacement for three
Table 1 Material properties of loading mechanism and sample

\begin{tabular}{lll}
\hline & Brass (sample) $^{\mathrm{a}}$ & Ti-6Al-4V (MLM) $^{\mathrm{b}}$ \\
\hline Young's modulus (GPa) & 112 & 113.8 \\
Poisson's ratio & 0.346 & 0.342 \\
Yield stress (MPa) & 204 & 880 \\
\hline
\end{tabular}

a Determined from uni-axial tensile experiments

b Boyer et al. (1994)

different loading positions for a specimen with a $6 \mathrm{~mm}$ long pre-crack. It can be observed that the mode angle stays almost constant with a maximum difference of $4^{\circ}$ between the minimum and maximum values of the three considered load cases for position $\xi=0.75$. Figure 9c shows the mode angle as a function of the crack length for the same three loading positions at $400 \mu \mathrm{m}$ displacement. The mode angle stays nearly constant for different crack lengths with a maximum difference of $2^{\circ}$ between the extreme values for position $\xi=0.75$. The simulations confirm that the new MMMB setup provides access to the complete range of mode angles whereby the mode angle does not change significantly with the applied displacement and/or evolving crack length.

\section{Validation}

The performance of the MMMB device is assessed in order to determine the influence of potential inaccuracies that may result from the geometry, machine 
Fig. 9 Mode angle obtained by FEM analysis as a function of (a) relative loading position $(\xi)$ for a crack length of $6 \mathrm{~mm}$ at $400 \mu \mathrm{m}$ MLM

displacement, (b) displacement applied to MLM for three loading positions for a $6 \mathrm{~mm}$ crack length specimen, and (c) crack length for three loading positions at $400 \mu \mathrm{m}$ MLM displacement
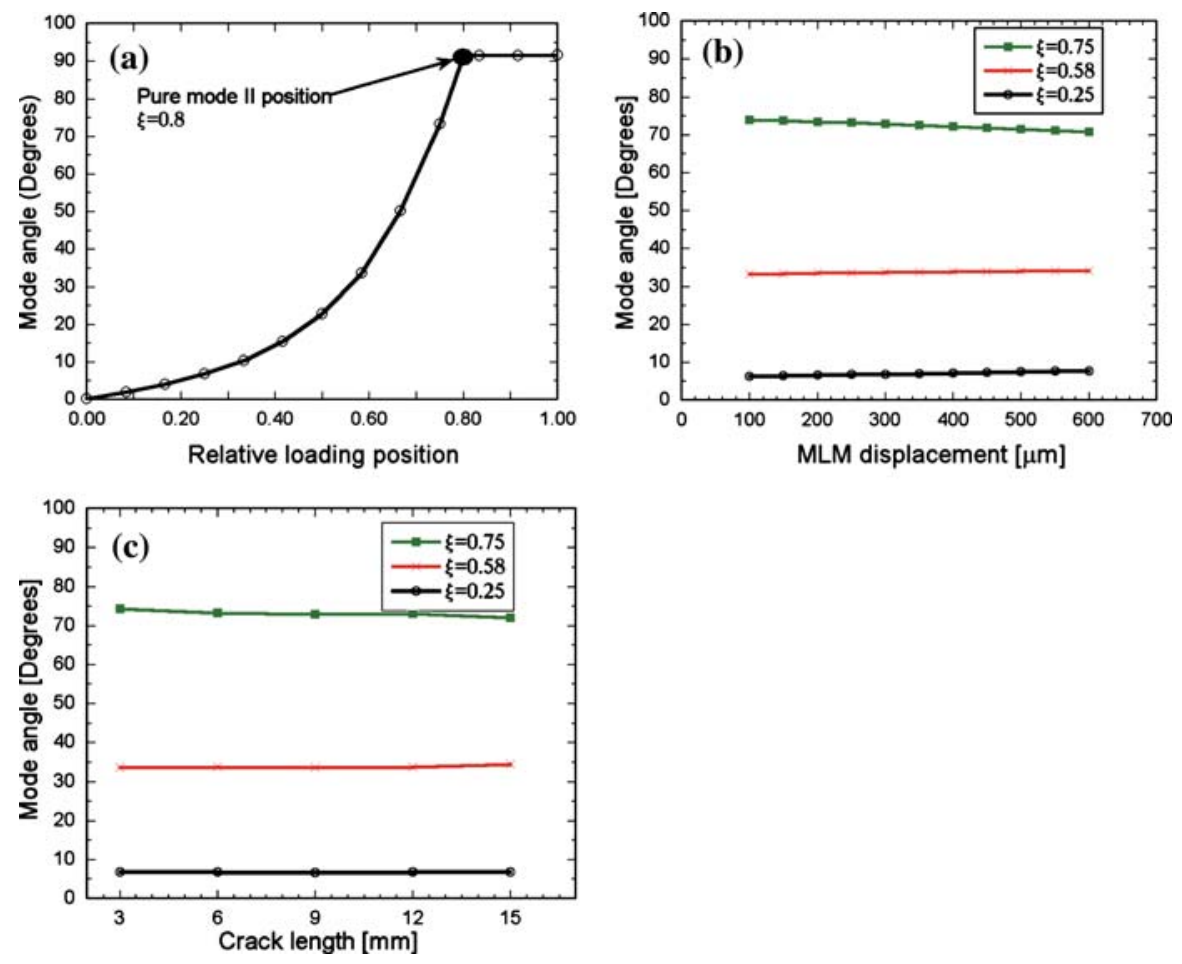

Fig. 10 End parts of (a) mode I/ mixed mode test sample and (b) mode II test sample. (c) Detailed image of the elastic beam of the mode II test sample

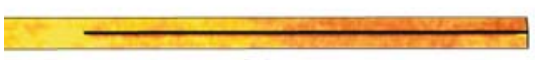

(a)

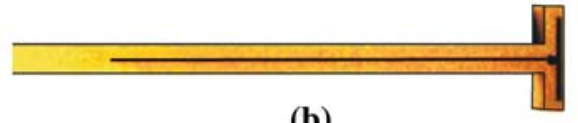

(b)

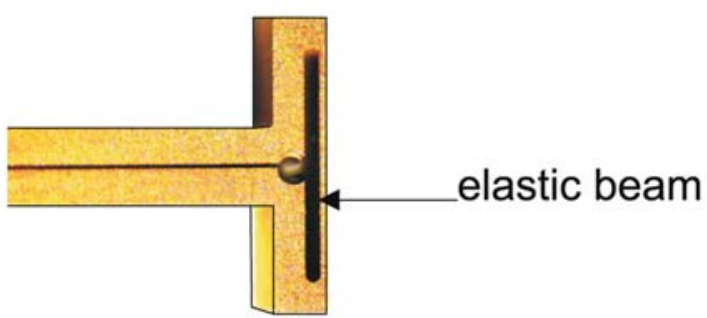

(c) compliance, or other possible factors like clearance at connectors. Specially designed test samples (shown in Fig. 10) have been used to this purpose. These are homogeneous, single layer brass samples (i.e. without an interface and no propagating crack), with a well defined notch, having an opening width of $30 \mu \mathrm{m}$, representing an existing crack of a fixed length. The thickness of these samples is $1 \mathrm{~mm}$. Figure 10 shows end portions of two different types of these test samples. The specimen shown in Fig. 10a is designed for mode I and mixed mode loading, and the specimen shown in Fig. 10b is designed for mode II loading with a verti- cal elastic beam at the end of the notch (see Fig. 10c). This special design is used in order to prevent contact between two arms of the notched portion in mode II tests $(\xi=0.8-1)$ where compressive mode I component is present.

Test samples with 5 different notch lengths $(3,6,9$, 12 and $15 \mathrm{~mm}$ ) were used to check the performance of the setup as a function of the notch length. Figure 11 shows the results of these five samples loaded at $\xi=0.5$. A small amount of hysteresis was identified as the result of some clearance in the dovetail connectors. The relative hysteresis, defined as the dis- 


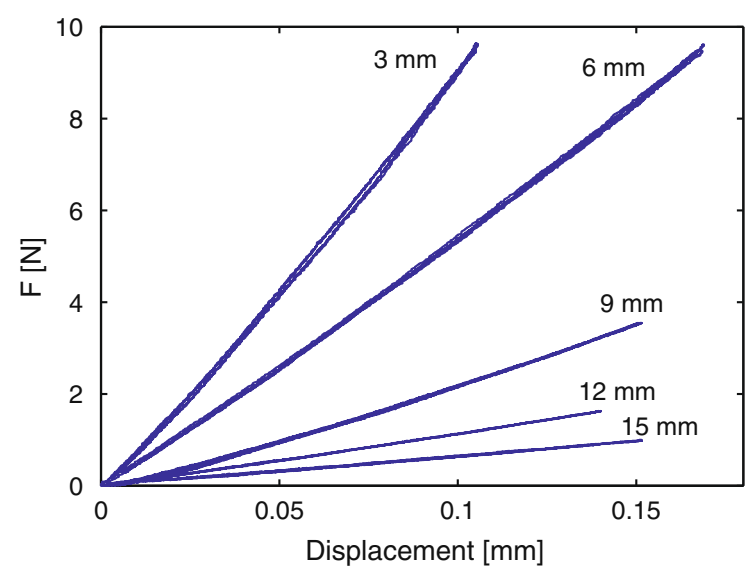

Fig. 11 Load-displacement results for loading-unloading cycles with $\xi=0.5$ for five crack lengths. The numbers indicate the crack length

sipated energy during a loading-unloading cycle, relative to the energy supplied during loading was calculated for all crack lengths and different loading positions. The maximum relative hysteresis was found to be $4 \%$ with a mean value of $3 \%$ for all positions between $\xi=0-0.8$. Comparison with digital image correlation (DIC) results confirmed that the hysteresis indeed originates from a minor clearance in the dovetail connectors. Even though the amount of hysteresis is limited, and not considered further in this paper, this will be improved in a future new connector design.

\section{Experimental results and discussion}

In-situ experiments with the new setup were conducted in a scanning electron microscope and under an optical stereo microscope at high magnifications. A batch of bilayer samples, consisting of two $0.3 \mathrm{~mm}$ thick spring steel layers glued together with an Araldite 2020 glue with a thickness of $\sim 5 \mu \mathrm{m}$ was used in the experiments. At the end of each sample, a pre-crack of $3 \mathrm{~mm}$ length was introduced with electro discharge machining. All the samples were heat treated at $80^{\circ} \mathrm{C}$ for 3 days to make the glue brittle and to fine tune the resulting glue strength. On the basis of these experiments, the interface properties of the custom made interface structure were characterized over a complete range of mode mixities, thereby demonstrating the practical functionality of the setup. Before the experiment, samples were fine polished on one side (perpendicular to the plane of the

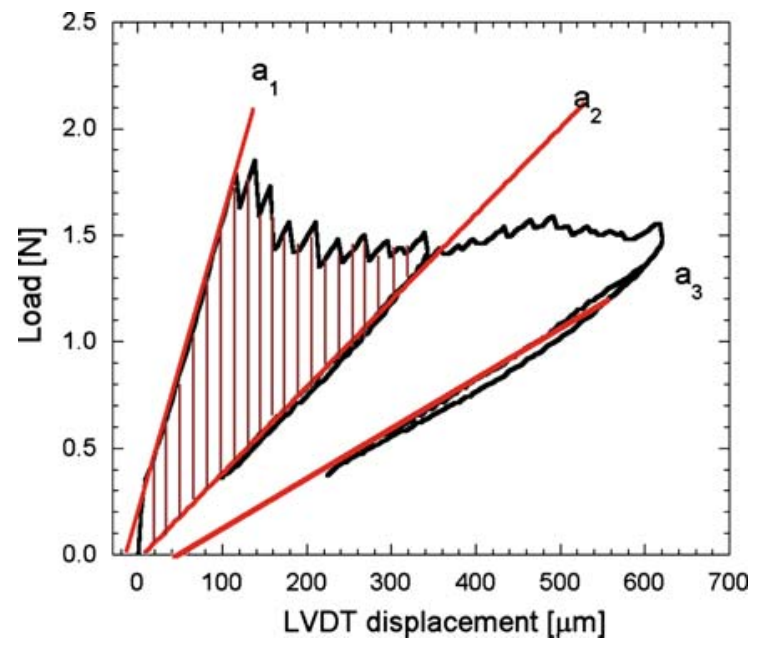

Fig. 12 Load-displacement response of a bilayer steel sample tested at position $\xi=0$. The stiffness lines are used to calculate the CERR

interface) to visualize the interface at high magnifications. Small markers with a regular spacing of approximately $500 \mu \mathrm{m}$ were introduced on the polished side with a sharp knife. These markers were used as a reference for tracking the position of the crack tip, since the crack tip moves out of the field of view of the microscope during the experiment. The dovetail connectors were attached on both sides of the samples with a strong glue at the pre-cracked end, after which the samples were carefully mounted into the setup.

Before actual loading of the specimen, each sample was loaded at position $\xi=0$ until the initiation of a sharp pre-crack was observed with a microscope. Finally, the position selector was reinserted at the appropriate position to carry out a delamination experiment at the desired mode angle. Actual loading was applied at a displacement rate of $3 \mu \mathrm{m} / \mathrm{s}$ and load and cross-head displacement values were recorded with the built in load cell $(20 \mathrm{~N})$ and linear variable differential transformer (LVDT) respectively. During the test, the sample was unloaded and reloaded at regular intervals. At each load reversal, images of the crack tip were recorded at a magnification of $250 \times$. These images were used to determine the crack length corresponding to the load reversals in the post processing analysis.

Figure 12 shows the load-displacement result of an in-situ experiment conducted under a stereo microscope, where the sample is loaded at position $\xi=$ 0 . Initially, at the beginning of the experiment, the 


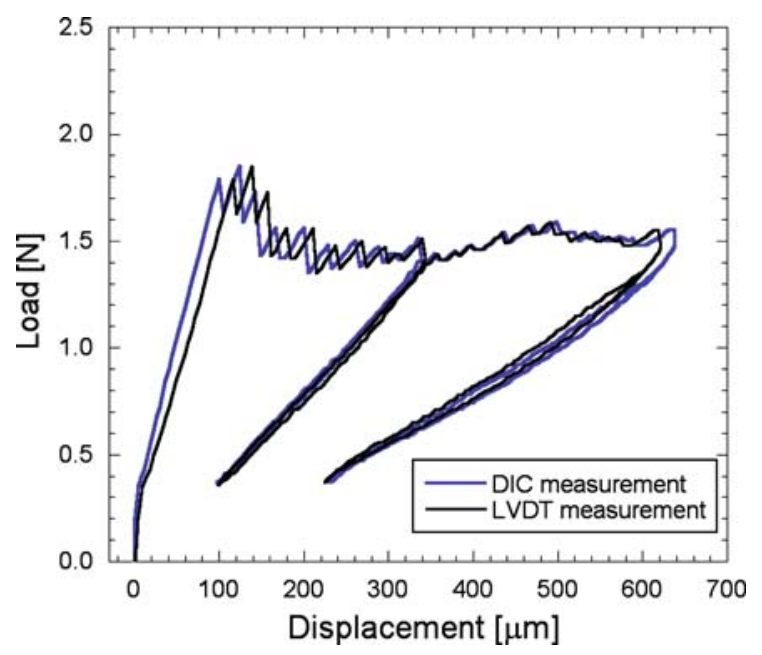

Fig. 13 Comparison of the raw load-displacement curves based on LVDT and DIC displacements

load increases rapidly with the displacement until the pre-crack starts propagating further. During this stage, the load remains approximately constant with small discontinuities with increasing displacement. Through real-time optical and scanning electron microscopic visualization, it is observed that the interface is delaminating in a discrete fashion. The observed serrated behavior is attributed to the discrete crack growth caused by non-uniformity of the adhesion strength over the interface area. This is confirmed by the microscopic observation which shows that, sometimes, the crack front jumps from one interface to the other. Apart from that, Fig. 12 also shows a small amount of hysteresis during each unloading-loading cycle.

In order to further verify that the observed (serrated and hysteresis) behavior originates from the interface and not from the setup, a separate displacement measurement is done using a digital image correlation (DIC) technique (Chu et al. 1985). Vertical opening displacements are measured by tracking the center points of the dovetail connectors on both sides of the sample, from a series of pictures taken during the delamination test, with the image correlation software Aramis. Comparison of LVDT and DIC based displacements, shown in Fig. 13, reveals an adequate agreement between these two measurements. An important observation is that the DIC measurement also shows the small hysteresis observed during the unloading-loading cycle with the LVDT measurement. This indicates that the hysteresis observed originates from the interface behav-

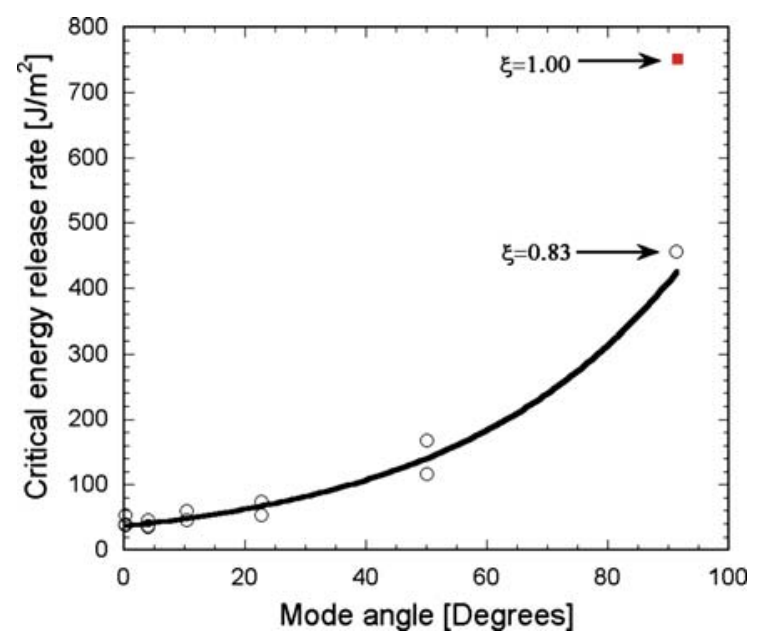

Fig. 14 CERR plotted as a function of mode angle. At $90^{\circ}$ mode angle the CERR measured for position $\xi=0.83$ is much lower than for position $\xi=1$

ior and that this effect is indeed captured by the new setup. A relatively small clearance $(15 \mu \mathrm{m})$ in the dovetail connectors explains the small deviation between both measurements, particularly in the initial portions of the curves. From the above discussion, it can be concluded that, (i) the new MMMB setup accurately captures the behavior of the interface and (ii) small deviations are present in the measured displacement due to some of clearance in the dovetail connections, which will have only a minor influence on the measured interface toughness.

Pictures taken during the load reversals were used to determine the precise crack lengths at each load reversal. Stiffness lines were fitted to the loading curves as shown in Fig. 12. The critical energy release rate of the interface was calculated from the area between the successive stiffness lines (the hashed region in Fig. 12), divided by the delaminated area corresponding to an increase of the crack length from position $a_{1}$ to $a_{2}$. The resulting CERR values are shown as a function of the mode angle, as determined from FE simulations, in Fig. 14. From this figure, it is clear that the CERR increases with increasing mode angle (towards position $\xi=1)$. It is noted that the CERR reported in this study represents the macroscopic interfacial fracture energy, which may include significant contributions from other dissipative mechanisms like plasticity in the layers apart from the intrinsic fracture energy. This is particularly the case when a ductile layer is present in the composite stack (Lane et al. 2000; Strohband 
Fig. 15 SEM micrographs showing the mechanism of delamination: bridging of the small cracks into a main crack. a Before crack evolution; b After crack evolution

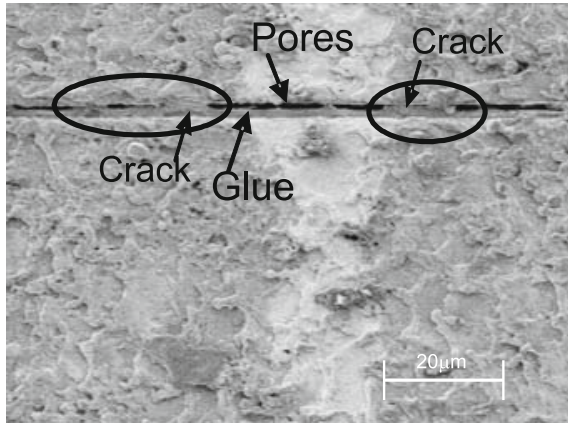

(a)

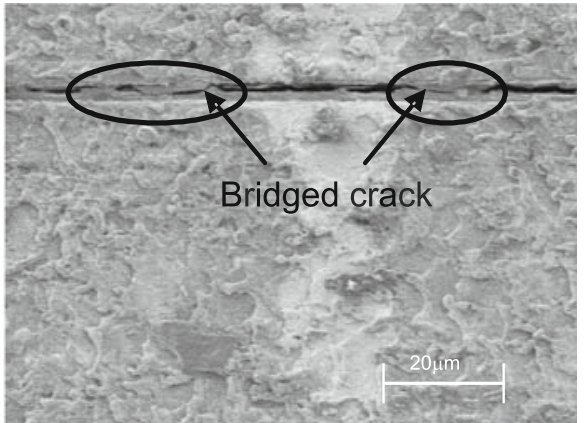

(b)

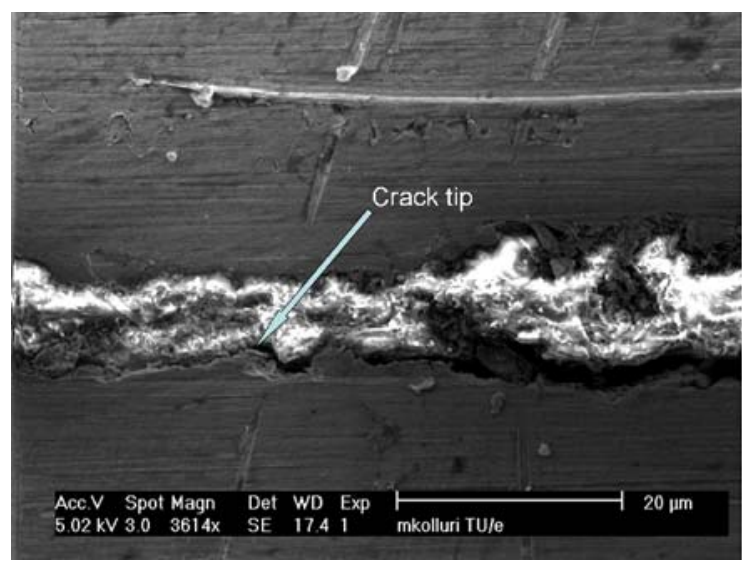

Fig. 16 SEM micrograph showing the precise crack tip location

and Dauskardt 2003). However, in the present study, the samples were heat treated for the specific reason to make the glue layer brittle which was confirmed from in-situ electron microscopy observation. Therefore, the plastic contributions to the CERR were minimized and hence neglected in this analysis. Another important observation from this figure is that a large difference exists between the CERR values measured at $\xi=0.83$ and at $\xi=1$. The position $\xi=0.83$ is close to pure mode II loading, and has a negligible compressive loading at the interface, whereas for the $\xi=1$ position (which resembles an ENF test), a compressive load is applied at the cracked portion of the two layers. The presence of this compressive load between the cracked layers leads to frictional dissipation causing an overestimation of the CERR values (Davies 1997). This result also indicates that to measure mode II delamination CERR, one needs to apply a pure mode II loading instead of a conventional ENF loading.
In-situ measurements performed in the SEM allowed to (i) visualize the delamination mechanism at the interface and (ii) identify the precise crack tip location. In Fig. 15, an interface with the crack growing from the right, is shown. Small pre-cracks of 50-100 $\mu \mathrm{m}$ appear in front of the crack tip before the actual crack evolution (Fig. 15a). These small pre-cracks grow and interconnect, resulting in the propagation of the main crack (Fig. 15b).

From the in-situ measurements, the position of the "crack tip" was determined with a n accuracy of $\sim 5 \mu \mathrm{m}$, see Fig. 16. In principle, this allows to extract the CERR more accurately than with conventional MMB setups. One remaining difficulty is tracking the crack tip as the delamination proceeds. This can be overcome by using special markers and interrupting the test to bring the crack tip location back into focus.

\section{Conclusion}

A new miniature setup capable of applying a mixed mode bending load to a bilayer delamination sample with a pre-crack was successfully introduced. The new setup can be used for in-situ delamination tests in advanced microscopic systems (e.g. SEM). The setup was designed with elastic hinges to overcome hysteresis due to friction. Comparison of the load-displacement diagrams from LVDT and DIC measurements showed that the present setup can accurately capture the interface behavior. An analysis of the setup proved the capability of the new setup to achieve a full range of mode mixities, with a nearly constant mode angle as a function of the crack length and crack opening displacement. The analysis also revealed that the conventional ENF test is not a true representation of a mode II delam- 
ination. The present setup can also apply a pure mode II loading (different from ENF loading) to obtain representative CERR values. The CERR measured for an ENF test is considerably higher than for a pure mode II loading, which was attributed to frictional dissipation between the two cracked surfaces. Finally, an in-situ test done in a SEM showed a high-resolution observation of the delamination mechanism. In addition, these in-situ tests allow for a more precise determination of the location of the crack tip.

Acknowledgements This research was carried out under the project number MC2.05235 in the framework of the Research Program of the Materials innovation institute M2i (http://www. m2i.nl), the former Netherlands Institute for Metals Research. Authors are grateful to M.P.F.H.L. van Maris for his assistance in conducting the experiments reported in this article.

Open Access This article is distributed under the terms of the Creative Commons Attribution Noncommercial License which permits any noncommercial use, distribution, and reproduction in any medium, provided the original author(s) and source are credited.

\section{References}

ASTM-D-5528-01 (2001) Standard test method for mode I interlaminar fracture toughness of unidirectional fiberreinforced polymer matrix composites. Annual book of ASTM standards, vol 15.03. American Society for Testing and Materials, West Conshohocken, PA

ASTM-D-6671-01 (2001) Standard test method for mixed mode I-mode II interlaminar fracture toughness of unidirectional fiber reinforced polymer matrix composites. Annual book of ASTM standards, vol 15.03. American Society for Testing and Materials, West Conshohocken, PA

Banks-Sills L, Travitzky N, Ashkenazi D (2000) Interface fracture properties of a bimaterial ceramic composite. Mech Mater 32:711-722

Banks-Sills L, Boniface V, Eliasi R (2005) Development of a methodology for determination of interface fracture toughness of laminate composites - the $0^{0} / 90^{0}$ pair. Int J Solids Struct 42:663-680

Bing Q, Sun CT (2007) Effect of compressive transverse normal stress on mode II fracture toughness in polymeric composites. Int J Fract. 145:89-97

Blanco N, Gamstedt E, Costa J, Trias D (2006) Analysis of the mixed-mode end load split delamination test. Compos Struct 76:14-20

Boyer R, Welsch G, Collings EW (1994) A materials properties hand book: titanium alloys. ASM international

Carlsson L, Gillespie J, Pipes J (1986) On the analysis and design of the end notched flexture specimen for mode II testing. J Compos Mater 20:594-604

Chu TC, Ranson WF, Sutton MA (1985) Applications of digital-image-correlation techniques to experimental mechanics. Exp Mech 25(3):232-244
Dauskardt RH, Lane M, Ma Q, Krishna N (1998) Adhesion and debonding of multi-layer thin film structures. Eng Fract Mech 61:141-162

Davidson B, Sundararaman V (1996) A single leg bending test for interfacial fracture toughness determination. Int J Fract 78:193-210

Davies P (1997) Influence of ENF specimen geometry and friction on the mode II delamination resistance of carbon/PEEK. J Thermoplast Compos Mater 10(4):353-361

Davies P, Blackman BRK, Brunner AJ (1998) Standard test methods for delamination resistance of composite materials: current status. Appl Compos Mater 5:345-364

Dreier G, Meyer M, Schmauder S, Elssner G (1992) Fracture mechanics studies of thermal mismatch using a four-point bending specimen. Acta Metall Mater 40:S345-S353

Evans A, Hutchinson J (1995) The thermomechanical integrity of thin films and multilayers. Acta Metall Mater 43(7):2507-2530

Evans A, Ruhle M, Dalgleish B, Charalambides P (1990) The fracture energy of bimaterial interfaces. Mater Sci Eng A 126:53-64

Fan C, Jar PYB, Cheng JJR (2007) A unified approach to quantify the role of friction in beam-type specimens for the measurement of mode II delamination resistance of fibrereinforced polymers. Compos Sci Tech 67:989-995

Gunderson JD, Brueck JF, Paris AJ (2007) Alternate test method for interlaminar fracture toughness of composites. Int J Fract 143:273-276

Gupta V, Pronin A (1995) New technique to measure the toughness of thin-film interfaces. J Am Ceram Soc 78(5):13971400

Hu M, Evans A (1989) The cracking and decohesion of thin films on ductile substrates. Acta Metall 37(3):917-925

Hutchinson J, Suo Z (1992) Mixed mode cracking in layered materials. Adv Appl Mech 29:63-191

Kuhl A, Qu J (2000) A technique to measure interfacial toughness over a range of phase angles. J Electron Packag 122: $147-151$

Lane M, Dauskardt RH, Vainchtein A, Gao H (2000) Plasticity contributions to interface adhesion in thin-film interconnect structures. J Mater Res 15(12):2758-2769

Li W, Siegmund T (2004) An analysis of the indentation test to determine the interface toughness in a weakly bonded thin film coating — substrate system. Acta Mater 52:2989_ 2999

Liu YF, Kagawa Y, Evans A (2008) Analysis of a barb test for measuring the mixed-mode delamination toughness of coatings. Acta Mater 56:43-49

Merrill CC, Ho PS (2004) Effect of mode mixity and porosity on interface fracture of low-k dielectrics. In: Materials research society symposium proceedings, vol 812. Paper: 5.7, Warrendale, PA

Ocaña I, Molina-Aldareguia J, Gonzalez D, Elizalde M, Sánchez J, Marti-nez-Esnaola J, Sevillano JG, Scherban T, Pantuso D, Sun B, Xu G, Miner B, He J, Maiz J (2006) Fracture characterization in patterned thin films by cross-sectional nanoindentation. Acta Mater 54:3453-3462

Reeder JR, Crews JR (1990) Mixed mode bending method for delamination testing. AIAA J 28(7):1270-1276

Shi X, Zhang X, Pang J (2006) Determination of interface fracture toughness of the adhesive joint subjected to 
mixed-mode loading using finite element method. Int J Adhes Adhes 26:249-260

Smith ST, Badami VG, Dale JS, Xua Y (1997) Elliptical flexure hinges. Rev Sci Instrum 68(3):1474-1483

Soboyejo W, Lu GY, Chengalva S, Zhang J, Kenner V (1999) A modified mixed-mode bending specimen for the interfacial fracture testing of dissimilar materials. Fatigue Fract Eng Mater Struct 22:799-810

Srikanth N, Chan L, Vath CJIII (2006) Adhesion improvement of EMC-leadframe interface using brown oxide promoters. Thin Solid Films 504:397-400

Strohband S, Dauskardt RH (2003) Interface separation in residually-stressed thin-film structures. Interface Sci 11:309317

Suo Z, Hutchinson J (1990) Interface crack between two elastic layers. Int J Fract 43:1-18

Tang S, Guo TF, Cheng L (2007) Rate dependent interface delamiantion in plastic IC packages. In: 9th Electronics packaging technology conference. Singapore, pp 680-685
Thijsse J, van der Sluis O, van Dommelen JAW, van Driel WD, Geers MGD (2008) Characterization of semiconductor interfaces using a modified mixed mode bending apparatus. Microelectron Reliab 48:401-407

Vinciquerra AJ, Davidson BD (2004) Effect of crack length measurement technique and data reduction procedures on the perceived toughness from four-point bend end-notched flexure tests. J Reinf Plast Compos 23:1051-1062

Vlassak JJ, Lin Y, Tsui TY (2005) Fracture of organosilicate glass thin films: environmental effects. Mater Sci Eng A 391:159-174

Volinsky A, Vella J, Gerberich W (2003) Fracture toughness, adhesion and mechanical properties of low-k dielectric thin films measured by nanoindentation. Thin Solid Films 429:201-210

Wang JA, Wright I, Lance M, Liu K (2006) A new approach for evaluating thin film interface fracture toughness. Mater Sci Eng A 426:332-345 